\title{
Características físico-químicas de cebolinhas comum e europeia
} Physicochemical characteristics of commom and european chives

\section{Autores | Authors}

\section{*Aline Priscilla Gomes da SILVA \\ Universidade Federal de Pelotas (UFPEL) Centro de Ciências Químicas Farmacêuticas e de Alimentos Programa de Pós-graduação em Ciência dos Alimentos Campus Universitário Caixa Postal: 354 CEP: $96010-900$ Pelotas/RS - Brasil \\ Universidade de São Paulo (USP) Escola Superior de Agricultura Luiz de Queiroz (ESALQ) \\ Departamento de Produção Vegetal Programa de Pós-graduação em Fitotecnia \\ Avenida Pádua Dias, 11, São Dimas CEP: 13418-900 \\ Piracicaba/SP - Brasil \\ e-mail: alinepgsilva@gmail.com}

\section{Caroline Dellinghausen BORGES}

Universidade Federal de Pelotas (UFPEL) Centro de Ciências Químicas,

Farmacêuticas e de Alimentos (CCQFA) Pelotas/RS - Brasil e-mail: caroldellin@bol.com.br

\section{Ana Carolina Almeida MIGUEL Angelo Pedro JACOMINO}

Universidade de São Paulo (USP) Escola Superior de Agricultura "Luiz de Queiroz" (ESALQ) Departamento de Produção Vegetal Piracicaba/SP - Brasil e-mail: anaamiguel@yahoo.com.br jacomino@usp.br

\section{Carla Rosane Barboza} MENDONÇA

Universidade Federal de Pelotas (UFPEL) Centro de Ciências Químicas, Farmacêuticas e de Alimentos (CCQFA) Pelotas/RS - Brasil e-mail: carlaufpel@hotmail.com

*Autor Correspondente / Corresponding Author Recebido: Maio 15, 2015 Aprovado: Nov. 30, 2015

\section{Resumo}

A cebolinha é uma folhosa bastante utilizada como condimento no Brasil, onde as espécies popularmente conhecidas como comum e europeia são as mais utilizadas. Objetivou-se com este trabalho avaliar a qualidade físico-química de cebolinhas Allium fistulosum (comum) e Allium schoenoprasum (europeia). As amostras das diferentes espécies foram analisadas quanto a: coloração (luminosidade, ângulo hue e cromaticidade); sólidos solúveis (SS); acidez titulável (AT); relação SS/AT; pH; umidade; clorofila a; clorofila b; clorofila total e carotenoides totais. Com relação aos parâmetros de cor, as espécies mostraram resultados muito próximos, evidenciando coloração característica de cebolinha, expressa pela cor verde com tendência ao amarelo. Também em relação ao pH observou-se semelhanças, enquadrando-se na faixa de baixa acidez. Já para SS e acidez, a variedade europeia apresentou maiores teores. Encontrou-se também proximidade de valores para os pigmentos avaliados, sendo a proporção de clorofila a em relação à $b$ em média de 2,3:1. Os resultados físico-químicos mostraram semelhanças entre as espécies, sendo observados baixa acidez, elevado conteúdo de umidade e de clorofila, além de razoáveis teores de sólidos solúveis e carotenoides. Essas informações sobre cebolinhas verdes cultivadas no Brasil, ainda escassas na literatura, vêm a contribuir com a produção de dados sobre essa hortaliça, podendo motivar seu consumo e a expansão de sua comercialização em formas diversificadas.

Palavras-chave: Allium fistulosum; Allium schoenoprasum; Qualidade pós-colheita; Cebolinha comum; Cebolinha europeia.

\section{Summary}

Chives are a leafy vegetable widely used as a condiment in Brazil, where the most used species are popularly known as common and European. The aim of this study was to evaluate the physicochemical quality of in natura Allium fistulosum (common) and Allium schoenoprasum (European) chives. The samples of the different species were harvested and analysed for colour (brightness, hue angle and chromaticity); soluble solids (SS); titratable acidity (TA); SS/TA; pH; moisture; chlorophyll $a$; chlorophyll $b$; total chlorophyll and total carotenoids. Regarding the colour parameters, the species showed similar results, with the characteristic colour of chives, expressed by the colour green with a tendency to yellow. The $\mathrm{pH}$ values were also similar, both fitting into the low acid range. However for SS and acidity, the European variety showed higher levels. The values found for the pigments evaluated were also similar, the average ratio of chlorophyll a:b being 2.3:1. The physicochemical data also showed similarities, both species presenting low acidity, high moisture and chlorophyll contents, and reasonable levels of soluble solids and carotenoids. This information about the chives grown in Brazil, still scarce in the literature, contributes to the production of data about this plant and could motivate its consumption and the expansion of its marketing in diverse ways.

Key words: Allium fistulosum; Allium schoenoprasum; Postharvest quality; Common chives; European chives. 


\section{Introdução}

A cebolinha é uma planta pertencente à família das Aliáceas, comumente utilizada como condimento. Sua durabilidade na pós-colheita é muito curta, devido, principalmente, à alta taxa metabólica e ao alto teor de água (FREDDO et al., 2014). Dentre as espécies, destaca-se a Allium fistulosum, originária da Sibéria, conhecida como cebolinha comum. A planta é considerada perene, apresenta folhas cilíndricas e fistulosas, com 300 a 500 mm de altura, coloração verde-escura, produz pequenos bulbos cônicos, envolvidos por uma película rósea, com perfilhamento e formação de touceira (ZÁRATE; VIEIRA, 2004).

A Allium schoenoprasum, originária da Europa e conhecida como cebolinha europeia, apresenta folhas finas e forma tufos bem fechados. Na base das hastes há formação de engrossamento semelhante a bulbos ovais, as folhas são numerosas, finas, verde-escuro, parecidas com as das gramíneas, mas são ocas, como as de cebola. As hastes florais têm 150 a 200 mm de altura. Tanto a cebolinha comum como a europeia são amplamente cultivadas no Brasil e estão presentes em quase todos os lares brasileiros (BATISTA, 2012; ZÁRATE et al., 2007).

A cor, um dos primeiros atributos avaliados pelo consumidor, é determinante para a aquisição do produto, exercendo forte influência na qualidade, sendo indicativa do estádio de maturação. Além de servir de estímulo aos consumidores, também se correlaciona com a função nutricional, estando associada aos teores de clorofila (CUI et al., 2004; KASIM et al., 2008).

A clorofila é o pigmento natural mais abundante na natureza e ocorre em quase todas as plantas. O seu conteúdo nas folhas é influenciado por diversos fatores bióticos e abióticos, sendo relacionado com a atividade fotossintética (AMARANTE et al., 2008; TAIZ; ZEIGER, 2013). A clorofila a e a $b$ são as principais formas desse pigmento, tipicamente encontradas em plantas superiores comumente utilizadas para a alimentação, ocorrendo em proporções aproximadas de 2,5 a 3,5:1 (STREIT et al., 2005).

Os carotenoides são pigmentos que contribuem para a cor amarela, vermelho ou laranja, sendo sintetizados por plantas superiores, bactérias e fungos (CUI et al., 2004). O teor de carotenoides nos órgãos vegetais varia bastante: por ocorrerem associados à clorofila, esses pigmentos geralmente encontram-se em quantidades apreciáveis em vegetais verdes (RODRIGUEZ-AMAYA; KIMURA, 2004). Os carotenoides podem desempenhar função antioxidante e têm demonstrado ação protetora contra a carcinogênese, tanto em estudos in vitro como in vivo, com animais e humanos (GOMES, 2007). A proteção das células contra os radicais livres, proporcionada por esses compostos, atribui a alguns deles alegações de propriedades funcionais (ANVISA, 2015).

Outros parâmetros que são avaliados na pós-colheita de produtos hortícolas, como a cebolinha, são: sólidos solúveis, acidez titulável e pH, que estão diretamente correlacionados com as características intrínsecas, sendo indicativos da conservação e vida útil. Constituem os sólidos solúveis os ácidos, os sais, as vitaminas hidrossolúveis, os aminoácidos, algumas pectinas e os açúcares presentes nos vegetais. Em geral, esses compostos são responsáveis pelo sabor e pela consequente aceitação por parte dos consumidores, sendo a determinação comumente realizada para indicar o grau de maturação desses produtos (LIMA et al., 2001).

A acidez titulável expressa a quantidade de ácidos orgânicos presente em alimentos e influencia sabor, odor, cor, estabilidade e manutenção de qualidade (CECCHI, 2003). Juntamente com a acidez, faz-se necessária a determinação do pH, que é um parâmetro indicativo da susceptibilidade ao desenvolvimento de micro-organismos e também associado a palatabilidade, já que correlaciona-se inversamente com a acidez. Alguns trabalhos têm descrito a importância da quantificação dessas variáveis físico-químicas em hortaliças folhosas (BRUECKNER; PERNER, 2006; MIGUEL; DURIGAN, 2007; REIS et al., 2014; SANTOS et al., 2014; VIÑA; CERIMELE, 2009).

Em relação às cebolinhas verdes, são poucos os trabalhos encontrados na literatura dedicados a avaliar suas características físico-químicas ou mesmo a estabilidade do produto, principalmente, de cultivo brasileiro. Destacam-se os estudos de Santos et al. (2014), Junqueira-Gonçalves et al. (2012), Frezza et al. (2011), Kopsell et al. (2010), Viña e Cerimele (2009), Kasim et al. (2008), Imahori et al. (2007), Miguel e Durigan (2007), Brueckner e Pernev (2006) e Cui et al. (2004), nos quais foram avaliados produtos procedentes de Portugal, Chile, Argentina, Estados Unidos, Argentina, Turquia, Japão, Brasil e China.

Diante do exposto, objetivou-se com este estudo determinar alguns parâmetros físico-químicos associados à qualidade em cebolinhas verdes das espécies Allium fistulosum (comum) e Allium schoenoprasum (europeia) cultivadas em Piracicaba, SP, Brasil.

\section{Material e métodos}

Cebolinhas, Allium fistulosum (comum) e Allium schoenoprasum (europeia), foram colhidas, em junho de 2014, em propriedade particular localizada no município de Piracicaba, SP (latitude: $22^{\circ} 43^{\prime} 31^{\prime \prime}$ e longitude: $\left.47^{\circ} 38^{\prime} 57^{\prime \prime}\right)$. A colheita foi realizada manualmente, quando atingiram completa maturação, definida com base na coloração das hastes, sendo elas em seguida cuidadosamente transportadas ao Laboratório de 
Pós-Colheita de Produtos Hortícolas (LPV-ESALQ-USP), em Piracicaba,SP. Executou-se a seleção quanto à ausência de defeitos, visando obter um lote uniforme, e analisou-se quanto à coloração - em colorímetro Minolta CR 300, expressando-se os resultados em luminosidade $\left(L^{*}\right)$, que varia de 100 (branco) a zero (preto), ângulo Hue, ou ângulo de cor $\left({ }^{\circ} \mathrm{h}\right)$, e cromaticidade $\left(\mathrm{C}^{*}\right)$, que expressa a pureza ou intensidade da cor (MINOLTA CORPORATION, 1994); acidez titulável (AT), de acordo com a metodologia descrita por Carvalho et al. (1990), sendo os resultados expressos em porcentagem de ácido pirúvico; teor de sólidos solúveis (SS), em refratômetro digital Atago PR-101, Palette, sendo os resultados expressos em ${ }^{\circ} \mathrm{Brix}$; $\mathrm{pH}$, em potenciômetro digital; umidade, com os resultados expressos em porcentagem; relação SS/ATT. Os parâmetros SS, pH e umidade foram determinados segundo metodologia descrita pela AOAC (HORWITZ, 2005).

Para a execução das análises físico-químicas as hastes foram trituradas e posteriormente analisadas. Determinou-se também o teor de clorofila a $(\lambda=663 \mathrm{~nm})$; clorofila $b(\lambda=646 \mathrm{~nm})$; clorofila total; e carotenoides totais $(\lambda=470 \mathrm{~nm})$, de acordo com a metodologia descrita por Lichtenthaler (1987), utilizando-se um espectrofotômetro Biochroom Libra S22, sendo os resultados expressos em $\mathrm{mg} \mathrm{g}^{-1}$.

As análises físicas de coloração foram realizadas em um espaço amostral de 50 repetições para ambas as espécies, enquanto as análises físico-químicas foram realizadas em amostras compostas das hastes e avaliadas em triplicata.

Os dados foram submetidos à análise de variância e a comparação de médias foi realizada pelo teste $F$, ao nível de $5 \%$ de probabilidade, por meio do programa SAS 7.0.

\section{Resultados e discussão}

A luminosidade $\left(L^{*}\right)$ em ambas as espécies mostrou valores médios de 43 , demonstrando que ambas apresentavam-se opacas (Tabela 1). Frezza et al. (2011), quando avaliaram cebolinhas recém-colhidas na Argentina, relataram valores de 40, ou seja, semelhantes aos encontrados no presente estudo. Santos et al. (2014), avaliando diferentes condimentos aromáticos cultivados em Portugal, encontraram valores de luminosidade de 30 , evidenciando que suas amostras eram mais claras do que as avaliadas neste trabalho, oriundas de Piracicaba, SP.

Os valores de ângulo hue indicaram que ambas as espécies apresentaram coloração característica de cebolinha, expressa pela cor verde com tendência ao amarelo, sendo os valores encontrados semelhantes aos descritos por Kasim et al. (2008) para hortaliças cultivadas na Turquia (ângulo hue $=105$ ).
No entanto, Imahori et al. (2007) encontraram valores de hue na ordem de 122, demonstrando que suas amostras de cebolinhas verdes plantadas no Japão apresentaram uma coloração amarelada mais acentuada. A cromaticidade define a pureza ou intensidade da cor. Verificou-se que as espécies de cebolinhas avaliadas apresentavam tendência à neutralidade, tendo em vista os baixos valores obtidos. Os valores de cromaticidade variam de 0 , para cores neutras, a 60, para cores vivas, ou seja, altos valores estão associados a maior intensidade da cor e os baixos, à neutralidade (MCGUIRE, 1992).

Avaliações de cor e suas alterações ao longo de tempo de armazenamento de cebolinhas foram reportadas por Junqueira-Gonçalves et al. (2012) e Viña e Cerimele (2009) em material cultivado no Chile e na Argentina, respectivamente. Junqueira-Gonçalves et al. (2012) observaram que a cor mostrou tendência ao amarelo em cebolinhas armazenadas a $4{ }^{\circ} \mathrm{C}$ durante 10 dias, obtendo para ângulo hue 19,89 no primeiro dia e 28 no décimo. Também Viña e Cerimele (2009), ao analisarem o ângulo hue e a luminosidade ao final de um período de 14 dias, obtiveram comportamento similar para cor, com valores de 135,2 e 38,2, respectivamente.

Os teores de SS (Tabela 2) da cebolinha europeia apresentaram diferenças significativas quando comparados com os da espécie comum. Santos et al. (2014) reportaram teores de 4,6 ${ }^{\circ}$ Brix para a Allium schoenoprasum (europeia), inferiores aos apresentados nesse trabalho, que foram de 5,2 ${ }^{\circ}$ Brix. O conteúdo de SS pode ser utilizado para a determinação do grau de maturação, representando um critério útil para estabelecer a estabilidade de produtos hortícolas ao armazenamento. No entanto, em alguns produtos, sua medição isolada pode induzir a erros,

Tabela 1. Parâmetros de coloração de cebolinhas comum e europeia oriundas da cidade de Piracicaba, SP, 2014.

\begin{tabular}{lccc} 
Cebolinha & $\mathbf{L}^{*}$ & ${ }^{\mathbf{h}}$ & $\mathbf{C}^{*}$ \\
Comum & $43,99^{\mathrm{a}}$ & $105,43^{\mathrm{a}}$ & $8,48^{\mathrm{a}}$ \\
Europeia & $43,77^{\mathrm{a}}$ & $103,58^{\mathrm{a}}$ & $8,01^{\mathrm{a}}$ \\
CV $(\%)$ & 2,32 & 3,65 & 8,10 \\
\hline
\end{tabular}

Médias seguidas de letras iguais nas colunas não diferem entre si, pelo teste F, em até $5 \%$ de probabilidade.

Tabela 2. Características físico-químicas de cebolinha comum e da europeia oriundas da cidade de Piracicaba, SP, 2014.

\begin{tabular}{|c|c|c|c|c|c|}
\hline Cebolinha & $\begin{array}{c}\text { Sólidos } \\
\text { solúveis } \\
\text { ( }{ }^{\circ} \text { Brix) }\end{array}$ & $\begin{array}{c}\text { Acidez } \\
\text { titulável } \\
\left(\text { g } 100 \mathbf{g ~ g}^{-1}\right)\end{array}$ & $\begin{array}{c}\text { Relação } \\
\text { SS/AT }\end{array}$ & pH & $\begin{array}{c}\text { Umidade } \\
(\%)\end{array}$ \\
\hline Comum & $4,65^{b}$ & $0,09^{a}$ & $47,77^{a}$ & $5,46^{a}$ & $88,82^{a}$ \\
\hline Europeia & $5,20^{a}$ & $0,12^{a}$ & $40,80^{a}$ & $5,46^{a}$ & $89,94^{a}$ \\
\hline CV (\%) & 1,02 & 8,17 & 8,78 & 2,96 & 1,50 \\
\hline
\end{tabular}

Médias seguidas de letras iguais nas colunas não diferem entre si, pelo teste F, ao nível de $5 \%$ de probabilidade. 
já que os níveis de pungência e sólidos solúveis estão diretamente relacionados com o estádio de maturação e são indicativos de susceptibilidade a alterações (GRANGEIRO et al., 2008).

A acidez titulável apresentou teores médios de 0,09 g e 0,12 g de ácido pirúvico em 100 g, podendo elas serem consideradas hortaliças de baixa acidez.

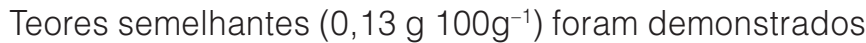
por Miguel e Durigan (2007) em bulbos de cebola (Allium cepa L.). Brueckner e Perner (2006) encontraram

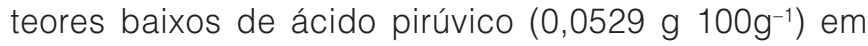
cebolinhas, corroborando os descritos neste trabalho. Apesar do maior valor médio da relação SS/AT encontrado para a cebolinha comum, a diferença não foi significativa. A evolução nos teores de açúcares solúveis e compostos ácidos podem provocar diferenças nas propriedades sensoriais do produto, especialmente na sensação de doçura e acidez (BARRETT et al., 2010).

Para o pH foram observados valores médios de 5,46 . Valores superiores $(6,01)$ foram encontrados por Santos et al. (2014) quando avaliaram cebolinha fresca da espécie europeia comercializada em Portugal. No caso da cebolinha foi verificada baixa acidez e alto pH (Tabela 2). Foram obtidos altos teores de umidade em ambas as espécies (Tabela 2). O elevado teor de umidade torna esse produto suscetível à perda de água e degradação da clorofila, com reflexos na redução de tamanho, massa, teor de sólidos solúveis e consequente redução de vida de prateleira (REIS et al., 2014).

Observou-se que as cebolinhas comum e europeia não diferiam em função do teor de clorofila (Tabela 3). Nas espécies estudadas, a relação entre as clorofilas a e b apresentaram em média a razão de 2,3:1 (Tabela 3). Os teores de clorofila total encontrados $\left(39,97 \mathrm{mg} \mathrm{g}^{-1}\right.$ e $33,67 \mathrm{mg} \mathrm{g}^{-1}$ ) foram muito superiores aos relatados por Cui et al. (2004), quando avaliaram cebolinhas comercializadas na China $\left(7,70 \mathrm{mg} \mathrm{g}^{-1}\right)$, bem como àqueles reportados por Frezza et al. (2011), que reportaram teor de clorofila de $26 \mathrm{mg} \mathrm{g}^{-1}$ em cebolinhas recém-colhidas na Argentina.

Em relação ao conteúdo de carotenoides totais também não houve diferença significativa entre as espécies, sendo os valores médios encontrados de 6,86 e $\mathrm{mg} \mathrm{g}^{-1} 6,33 \mathrm{mg} \mathrm{g}^{-1}$, respectivamente, superiores aos descritos por Egert e Tevini (2002), que detectaram teores de $1,3 \mathrm{mg} \mathrm{g}^{-1}$ em cebolinhas (Allium schoenoprasum L. cV Grobröhrig Hilds Polycross) produzidas na Alemanha. Kopsell et al. (2010), avaliando os teores desses pigmentos em diferentes acessos de Allium fistulosum L., procedentes dos Estados Unidos, encontraram entre 4,84 mg $100 \mathrm{~g}^{-1}$ a 6,03 mg $100 \mathrm{~g}^{-1}$, similares aos relatados no presente trabalho. A síntese de carotenoides relaciona-se inversamente com a de clorofila, o maior teor
Tabela 3. Teores de clorofila e carotenoides em cebolinhas comum e europeia oriundas da cidade de Piracicaba, SP, 2014.

\begin{tabular}{|c|c|c|c|c|}
\hline Cebolinha & $\begin{array}{c}\text { Clorofila } \\
\text { a } \\
\left(\mathbf{m g ~ g}^{-1}\right)\end{array}$ & $\begin{array}{c}\text { Clorofila } \\
\qquad b \\
\left(\mathrm{mg} \mathrm{g}^{-1}\right)\end{array}$ & $\begin{array}{c}\text { Clorofila } \\
\text { total } \\
\left(\mathbf{m g ~ g}^{-1}\right)\end{array}$ & $\begin{array}{c}\text { Carotenoides } \\
\text { totais } \\
\left(\mathrm{mg} \mathrm{g}^{-1}\right)\end{array}$ \\
\hline Comum & $27,62^{a}$ & $12,35^{a}$ & $39,97^{a}$ & $6,86^{a}$ \\
\hline Europeia & $23,82^{a}$ & $9,84^{a}$ & $33,67^{a}$ & $6,33^{a}$ \\
\hline CV (\%) & 10,54 & 23,84 & 14,42 & 18,19 \\
\hline
\end{tabular}

Médias seguidas de letras iguais nas colunas não diferem entre si, pelo teste F, ao nível de $5 \%$ de probabilidade.

de clorofila encontrado pode justificar o menor teor de carotenoides nesse produto.

Essas variações nos teores de clorofila e de carotenoides totais podem ser influenciadas pelas condições edafoclimáticas e práticas culturais empregadas (RESENDE et al., 2010).

A retenção de clorofila e carotenoides é muito importante para determinar a qualidade final das hortaliças verdes. As duas formas de clorofila são sensíveis a calor, oxigênio, luz, enzimas e ação de metais. A oxidação, principal causa de degradação dos carotenoides, depende da disponibilidade de oxigênio, do tipo de carotenoide e de seu estado físico. A degradação é estimulada por luz, calor, metais, enzimas oxidativas e peróxidos, sendo inibida por antioxidantes (MESCHEDE et al., 2011). Muito esforço tem-se empreendido para compreender a natureza das alterações de cor (CUI et al., 2004).

Vários estudos epidemiológicos indicaram que a alta ingestão de produtos vegetais está associada com uma redução no risco de uma variedade de doenças crônicas como aterosclerose e câncer. Esses efeitos têm sido atribuídos particularmente aos compostos que possuem atividade antioxidante. Os principais antioxidantes nos vegetais são as vitaminas $\mathrm{C}$ e $\mathrm{E}$, os carotenoides e os compostos fenólicos, especialmente os flavonoides. Já a clorofila tem mostrado efeitos benéficos à saúde por suas propriedades antimutagênicas e antigenotóxicas (LILA, 2004; SAINI et al., 2015; SILVA et al., 2010).

\section{Conclusão}

As cebolinhas comum e europeia apresentaram similaridade nas características avaliadas, mostrando ambas teores consideráveis de sólidos solúveis e baixa acidez. Essas variedades também apresentaram elevado teor de clorofila e teor razoável de carotenoides totais, compostos associados a benefícios à saúde humana.

As informações produzidas sobre cebolinhas verdes de cultivo brasileiro ainda são escassas na literatura, dessa forma, espera-se que o estudo venha a contribuir com dados sobre esse vegetal que motivem seu consumo, sua industrialização e a expansão de suas 
Características físico-químicas de cebolinhas comum e europeia

SILVA, A. P. G. et al.

formas comercialização no Brasil, a exemplo do vem ocorrendo a partir do processamento mínimo.

\section{Referências}

AGÊNCIA NACIONAL DE VIGILÂNCIA SANITÁRIA - ANVISA. Alegações de propriedade funcional aprovadas. Brasília, 2015. Disponível em: <http://portal.anvisa.gov.br/wps/content/ Anvisa+Portal/Anvisa/Inicio/Alimentos/Assuntos+de+Interesse/ Alimentos+Com+Alegacoes + de+Propriedades+Funcionais $+e$ + ou+de+Saude/Alegacoes+de+propriedade+funcional+apro vadas>. Acesso em: 15 set. 2015.

AMARANTE, C. V.; BISOGNIN, D. A.; STEFFENS, C. A.; ZANARDI, O. Z.; ALVES, E. D. O. Non-destructive quantification of chlorophylls in leaves by means of a colorimetric method. Horticultura Brasileira, Brasília, v. 26, n. 4, p. 471-475, 2008. http://dx.doi.org/10.1590/S0102-05362008000400009.

BARRETT, D. M.; BEAULIEU, J. C.; SHEWFELT, R. Color, flavor, texture, and nutritional quality of fresh-cut fruits and vegetables: desirable levels, instrumental and sensory measurement, and the effects of processing. Critical Reviews in Food Science and Nutrition, Boca Raton, v. 50, n. 5, p. 369-389, 2010. http:// dx.doi.org/10.1080/10408391003626322. PMid:20373184.

BATISTA, I. Cultivo da cebolinha. [S.I.]: Informática e Agricultura, 2012. Disponível em: <http://iuribatista.blogspot. com.br/2012/01/cultivo-da-cebolinha.html>. Acesso em: 14 mar. 2015.

BRUECKNER, B.; PERNER, H. Distribution of nutritive compounds and sensory quality in the leafs of chives (Allium schoenoprasum L.). Journal of Applied Botany and Food Quality, Germany, v. 80, n. 2, p. 155-159, 2006.

CARVALHO, C. R. L.; MANTOVANI, D. M. B.; CARVALHO, P. R. N.; MORAES, R. M. M. Análises químicas de alimentos. Campinas: ITAL, 1990. $121 \mathrm{p}$.

$\mathrm{CECCHI}, \mathrm{H}$. M. Fundamentos teóricos e práticos em análises de alimentos. 2. ed. Campinas: Editora da UNICAMP, 2003.

CUI, Z. W.; XU, S. Y.; SUN, D. W. Effect of microwavevacuum drying on the carotenoids retention of carrot slices and chlorophyll retention of Chinese chive leaves. Drying Technology, Philadelphia, v. 22, n. 3, p. 563-575, 2004. http:// dx.doi.org/10.1081/DRT-120030001.

EGERT, M.; TEVINI, M. Influence of drought on some physiological parameters symptomatic for oxidative stress in leaves of chives (Allium schoenoprasum). Environmental and Experimental Botany, Oxford, v. 48, n. 1, p. 43-49, 2002. http:// dx.doi.org/10.1016/S0098-8472(02)00008-4.

FREDDO, A. R.; CECHIM, F. E.; MAZARO, S. M. Conservation of post-harvest leaves of green onion (Allium fistulosum L.) with the use of salicylic acid solution. Brazilian Journal of Applied Technology for Agricultural Science, Paraná, v. 6, n. 3, p. 87-94, 2014.
FREZZA, D.; LEON, A.; LOGEGARAY, V.; CHIESA, A. Postharvest quality of green onion grown in soilless culture: effect of packaging and storage temperature. Agricultura Tropica et Subtropica, Czech Republic, v. 44, n. 1, p. 11-17, 2011.

GOMES, F. S. Carotenóides: uma possível proteção contra o desenvolvimento de câncer. Revista de Nutrição, Campinas, v. 20, n. 5, p. 537-548, 2007.

GRANGEIRO, L. C.; SOUZA, J. D. O.; AROUCHA, E. M. M.; NUNES, G. H. D. S.; SANTOS, G. M. Características qualitativas de genótipos de cebola. Ciência e Agrotecnologia, Lavras, V. 32, n. 4, p. 1087-1091, 2008.

HORWITZ, W. (Ed.). Official methods of analysis of the Association of Official Analytical Chemists. 18. ed. Gaithersburg: AOAC, 2005.

IMAHORI, Y.; SUZUKI, Y.; KAWAGISHI, M.; ISHIMARU, M.; UEDA, Y.; CHACHIN, K. Physiological responses and quality attributes of Chinese chive leaves exposed to $\mathrm{CO}$ 2-enriched atmospheres. Postharvest Biology and Technology, Amsterdam, v. 46, n. 2, p. 160-166, 2007. http://dx.doi. org/10.1016/j.postharvbio.2007.04.008.

JUNQUEIRA-GONÇALVES, M. P.; ZUNIGA, G. E.; ZÁRATE, H.; ARCOS, K.; GANGA, A.; MILTZ, J. Effect of y radiation on chives safety and quality. International Journal of Food Science \& Technology, Oxford, v. 47, n. 11, p. 2436-2443, 2012. http:// dx.doi.org/10.1111/j.1365-2621.2012.03120.x.

KASIM, M. U.; KASIM, R.; ERKAL, S. UV-C treatments on fresh-cut green onions enhanced antioxidant activity, maintained green color and controlled 'telescoping'. Journal of Food Agriculture \& Environment, Finland, v. 6, n. 3-4, p. 63-67, 2008.

KOPSELL, D. A.; SAMS, C. E.; DEYTON, D. E.; ABNEY, K. R.; KOPSELL, D. E.; ROBERTSON, L. Characterization of nutritionally important carotenoids in bunching onion. HortScience, Alexandria, v. 45, n. 3, p. 463-465, 2010.

LICHTENTHALER, H. K. Chorophylls and carotenoids: pigments of photosynthetic biomembranes. Methods in Enzymology, New York, v. 148, n. 22, p. 346-382, 1987.

LILA, M. A. Plant pigments and human health. In: DAVIS, S. Plant pigments and their manipulation. Oxford: Blackwell Publishing, 2004. p. 248-274.

LIMA, K. S. C.; GROSSI, J. L.; LIMA, A. L. S.; ALVES, P. F. M. P.; CONEGLIAN, R. C. C.; GODOY, R. L. O.; SABAA-SRUR, A. U. $O$. Efeito da irradiação ionizante g na qualidade póscolheita de cenouras (Daucus carota L.) cv. Nantes. Ciência e Tecnologia de Alimentos, Campinas, v. 21, n. 2, p. 202-208, 2001. http:// dx.doi.org/10.1590/S0101-20612001000200015.

MCGUIRE, R. D. Reporting of objective color measurements. HortScience, Alexandria, v. 27, n. 12, p. 1254-1255, 1992.

MESCHEDE, D. K.; VELINI, E. D.; CARBONARI, C. A.; SILVA, J. R. M. Alteração fisiológica da cana-de-açúcar pela aplicação 
de glyphosate e sulfumeturon-methyl. Planta Daninha, Viçosa, v. 29, n. 2, p. 413-419, 2011. http://dx.doi.org/10.1590/S010083582011000200019 .

MIGUEL, A. C. A.; DURIGAN, J. F. Qualidade de cebola minimamente processada e armazenada sob refrigeração. Horticultura Brasileira, Brasília, v. 25, n. 3, p. 437-441, 2007. http://dx.doi.org/10.1590/S0102-05362007000300022.

MINOLTA CORPORATION. Precise color communication: color control from feeling to instrumentation. Ramsey: Minolta Corporation Instrument Systems Division, 1994. 49 p.

REIS, H. F. M.; MELO, C.; MELO, E. P.; SILVA, R. A.; SCALON, S. P. Post-harvest conservation of crisp lettuce under modified atmosphere, cultivated on organic and conventional system. Horticultura Brasileira, Brasília, v. 32, n. 3, p. 303-309, 2014. http://dx.doi.org/10.1590/S0102-05362014000300011.

RESENDE, A. L. S.; VIANA, A. J. D. S.; OLIVEIRA, R. J.; AGUIARMENEZES, E. D. L.; RIBEIRO, R. D. L.; RICCI, M. D. S.; GUERRA, J. G. M. Consórcio couve-coentro em cultivo orgânico e sua influência nas populações de joaninhas. Horticultura Brasileira, Brasília, v. 28, n. 1, p. 41-46, 2010. http://dx.doi.org/10.1590/ S0102-05362010000100008.

RODRIGUEZ-AMAYA, D.; KIMURA, M. HarvestPlus handbook for carotenóides analysis. Washington: IFPRI; Cali: CIAT, 2004. 58 p. (HarvestPlus Technical Monograph, 2).

SAINI, R. K.; NILE, S. H.; PARK, S. W. Carotenoids from fruits and vegetables: chemistry, analysis, occurrence, bioavailability and biological activities. Food Research International, Oxford, v. 76, p. 735-750, 2015. http://dx.doi.org/10.1016/j. foodres.2015.07.047.
SANTOS, J.; HERRERO, M.; MENDIOLA, J. A.; OLIVA-TELES, M. T.; IBÁÑEZ, E.; DELERUE-MATOS, C.; OLIVEIRA, M. B. P. P. Fresh-cut aromatic herbs: nutritional quality stability during shelf-life. LWT-Food Science and Technology, London, v. 59, n. 1, p. 101-107, 2014. http://dx.doi.org/10.1016/j.Iwt.2014.05.019.

SILVA, M. L. C.; COSTA, R. S.; DOS SANTOS SANTANA, A.; KOBLITZ, M. G. B. Compostos fenólicos, carotenóides e atividade antioxidante em produtos vegetais. Semina: Ciências Agrárias, Londrina, v. 31, n. 3, p. 669-682, 2010.

STREIT, N. M.; CANTERLE, L. P.; CANTO, M. W. D.; HECKTHEUER, L. H. H. The chlorophylls. Ciência Rural, Santa Maria, V. 35, n. 3, p. 748-755, 2005. http://dx.doi.org/10.1590/S010384782005000300043.

TAIZ, L.; ZEIGER, E. Plant physiology. 5. ed. Sunderland: Sinauer Associates, 2013. 918 p.

VIÑA, S. Z.; CERIMELE, E. L. Quality changes in fresh chives (Allium schoenoprasum L.) during refrigerated storage. Journal of Food Quality, Spain, v. 32, n. 6, p. 747-759, 2009. http:// dx.doi.org/10.1111/j.1745-4557.2009.00281.x.

ZÁRATE, N. A. H.; VIEIRA, M. C.; BRATTI, R. Efeitos da cama-defrangos e da época de produção e a renda bruta da cebolinha "todo ano". Pesquisa Agropecuária Tropical, Goiás, v. 33, n. 2, p. 73-78, 2007.

ZÁRATE, N. A. H.; VIEIRA, M. C. Produção e renda bruta da cebolinha solteira e consorciada com espinafre. Horticultura Brasileira, Brasília, v. 22, n. 4, p. 811-814, 2004. http://dx.doi. org/10.1590/S0102-05362004000400031. 